\title{
El actual trabajo docente universitario en Argentina: alertas para repensar
}

\section{$O$ atual trabalho docente universitário na Argentina: alertas para repensar}

\section{The current university teaching work in Argentina: warnings to rethink about it}

\author{
María Alicia Villagra $^{1}$
}

\begin{abstract}
RESUMEN
Esta contribución refiere a la actual reconfiguración del trabajo del docente universitario argentino por el impacto de las políticas académicas de los 1990, principalmente por el Programa de Incentivos. Este Programa, propiciando la articulación entre docencia, investigación, extensión y gestión, legitima el pasaje de la tradicional figura del docente universitario dedicado a la enseñanza a la del docente-investigador. El aporte que presentamos se inscribe en avances del Proyecto de Investigación "Intensificación del trabajo docente y enseñanza universitaria: encuentros y desencuentros entre tiempos y espacios personales-profesionales", investigación cualitativa e interdisciplinaria radicada en la Facultad de Filosofía y Letras de la Universidad Nacional de Tucumán (Argentina), que se propuso estudiar el vínculo entre la intensificación del trabajo del docente, los procesos de colonización espacio-temporal de su ámbito privado por el profesional y sus repercusiones en la calidad de la enseñanza universitaria. Identificamos la emergencia de nuevas tensiones y exigencias enmarcadas en un escenario laboral hiper-evaluativo y sus resonancias en la redefinición identitaria del protagonista del trabajo académico, en la vida de las cátedras y en los modos de convivencia institucional. En suma, rescatando lo silenciado por los docentes frente a las políticas hegemónicas que jerarquizan la investigación sobre la docencia, sitúa a la enseñanza universitaria en "zona de riesgo" con la intencionalidad de compartir alertas para repensar.
\end{abstract}

Palabras-clave: trabajo docente; incentivos; identidad; políticas; enseñanza.

DOI: $10.1590 / 0104-4060.42594$

1 Universidad Nacional de Tucumán. Facultad de Filosofía y Letras. San Miguel de Tucumán, Argentina. Av. Benjamín Aráoz, nº 800. CP: 4000.E-mail: fliaburgos@infovia.com.ar 


\title{
RESUMO
}

Esta contribuição se refere à atual reconfiguração do trabalho docente universitário argentino em razão do impacto das políticas acadêmicas dos anos 1990, principalmente do Programa de Incentivos. Este Programa, ao propiciar a articulação entre docência, investigação, extensão e gestão, legitima a passagem da tradicional figura do docente universitário, dedicado ao ensino, para a figura do docente-pesquisador. $\mathrm{O}$ aporte que apresentamos inscreve-se nos avanços do Projeto de Pesquisa "Intensificação do trabalho docente e ensino universitário: encontros e desencontros entre tempos e espaços pessoais-profissionais", pesquisa qualitativa e interdisciplinar, realizada na Faculdade de Filosofia e Letras da Universidade Nacional de Tucumán (Argentina), que se propôs a estudar o vínculo entre a intensificação do trabalho do docente, os processos de colonização espaço-temporal do seu âmbito privado pelo profissional e suas repercussões na qualidade de ensino universitário. Identificamos a emergência de novas tendências e exigências contextualizadas num cenário de trabalho hiperavaliativo e suas ressonâncias na redefinição identitária do protagonista do trabalho acadêmico, na vida das diferentes disciplinas e nos modos de convívio institucional. Enfim, resgatando o que foi silenciado pelos docentes frente às políticas hegemônicas que hierarquizam a pesquisa sobre a docência, localiza o ensino universitário numa "zona de risco" com a intenção de compartilhar alertas para repensar.

Palavras-chave: trabalho docente; incentivos; identidade; políticas; ensino.

\begin{abstract}
This contribution refers to the current reshaping of the Argentine university professors' work due to the impact of the academic policies of the 1990s, particularly to the Program of Incentives. This Program, by promoting the articulation among teaching, research, extension and management, legitimizes the passage from the traditional figure of the university professor devoted to teaching to that of a professor-investigator. The contribution of our investigation is inscribed within the advances of the Research Project entitled "Intensification of teaching work and university teaching: agreements and misunderstandings between personal-professional time and space", a qualitative interdisciplinary investigation performed at the Faculty of Philosophy and Literature of the National University of Tucumán (Argentina) which intended to study the link between the intensification of teaching work, the processes of space and time colonization of its private setting by the professionals and its consequences on the university teaching quality. We identified the emergence of new paths and demands framed in a hyperevaluative labor scenario and their consequences on the identity redefinition of the protagonist of academic work, on the existence of chairs
\end{abstract}


and on the ways of interaction in an institutional atmosphere. In brief, rescuing the teachers' silence from the hegemonic policies that prioritize research over teaching, in which university teaching is located in a "risk zone", is for the purpose of sharing warnings to rethink about it.

Keywords: teaching work; incentives; identity; policies; teaching.

\section{El trabajo docente como objeto de investigación: despuntando reflexiones}

El trabajo docente en las universidades argentinas ha sufrido profundas modificaciones estructurales a partir de la instauración de las políticas académicas vigentes desde los 1990, entre las que cobra especial relevancia el Programa de Incentivos a los Docentes Investigadores de las Universidades Nacionales (Decreto $n^{\circ}$ 2427/93). Este Programa, creado por la Secretaría de Políticas Universitarias (SPU), se propone una reconversión de la planta docente promoviendo la articulación entre docencia, investigación, extensión y gestión. $\mathrm{Su}$ institucionalización impacta sobre la identidad del docente en tanto lo re-designa. De ser reconocido como docente a secas, un nuevo nombre lo resitúa frente a su tarea: el de docente-investigador; figura que imbrica dos funciones sustanciales, las de transmisión y la de producción del conocimiento, sin excluir, tal como lo señalamos, la de gestión y la de extensión.

El ingreso de esta figura en la escena universitaria alerta sobre síntomas que develan un complejo reacomodamiento laboral por parte de los docentes, hecho que afecta no sólo su profesionalidad sino su subjetividad y desafía agudos replanteos.

En la línea de estas inquietudes, el aporte que presentamos se inscribe en avances del Proyecto de Investigación denominado "Intensificación del trabajo docente y enseñanza universitaria: encuentros y desencuentros entre tiempos y espacios personales-profesionales" (CIUNT 24 H/444), avalado por la Secretaría de Ciencia y Técnica y radicado en la Facultad de Filosofía y Letras de la Universidad Nacional de Tucumán (UNT) Argentina. Aborda la incidencia de las regulaciones políticas en la reconfiguración del trabajo docente, centrándose en el análisis del vínculo entre la intensificación del trabajo del docente, los procesos de colonización espacio-temporal de su ámbito privado por el profesional y sus repercusiones en la calidad de la enseñanza universitaria. En suma, ausculta la dimensión subjetiva de este profesional de la enseñanza y 
registra su mirada e impresiones acerca de cómo piensa, siente y actúa frente a la normativa legalizada.

Este Proyecto, encarado por un equipo de carácter interdisciplinario y sustentado en una metodología de carácter cualitativa se propone, entre otros los siguientes objetivos:

- Discriminar efectos no deseados en la enseñanza universitaria originados por un docente que ante la intensificación de su trabajo se debate entre la calidad y la cantidad de actividades, entre el deseo y la factibilidad de cumplirlas y entre los cuándo y dónde satisfacerlas;

- Analizar las lógicas que regulan la interrelación entre tiempos y espacios subjetivos/objetivos del docente, a fin de identificar y caracterizar los puntos de fusión /invasión /confusión entre la esfera laboral y la privada;

- Otorgar visibilidad a modos idiosincrásicos de distribución y consumo de tiempos y espacios de trabajo, que más allá del aula e invisibles a la imagen pública de la docencia imponen ritmos y desplazamientos no siempre compatibles con las posibilidades profesionales/personales del docente;

- Identificar estrategias defensivas presentes en la enseñanza, desplegadas por el docente para soportar, negar o reparar sensaciones de impotencia e incompetencia frente a las exigencias académicas vivenciadas como intolerables sobreexigencias;

Como modalidad de abordaje, optamos por la utilización de diversas técnicas de obtención de información tales como mediaciones audiovisuales en estrategias de formación pedagógica, entrevistas en profundidad, grupos focales, análisis documental y técnicas narrativas.

En este trabajo nos basaremos en la información recabada a través de dos grupos focales en los que participaron docentes que cursan / cursaron carreras de posgrado en la UNT, con procesos de escritura de tesis en curso o a iniciar. Fueron seleccionados según un criterio de heterogeneidad, es decir de diferentes carreras, titulaciones, categorías, dedicaciones, franja etaria y sexo. Este material se complementó con entrevistas en profundidad a quienes, por sus intervenciones durante la instancia grupal, aportaron significativos textos $y$ pretextos como objeto de profundización. Adoptamos esta decisión metodológica por considerar que estos docentes-estudiantes representan el perfil sintetizador de la actual intensificación del trabajo docente, ya que, sin contar con el tiempo completo propio del alumnado de posgrado, deben compatibilizar el aumento de actividades implantadas a su función con la elaboración de sus trabajos de tesis.

Ahora bien, a los fines de caracterizar el inquietante escenario que enmarca las prácticas docentes universitarias, despuntaremos estas reflexiones haciendo referencia a las notas inherentes al Programa de Incentivos y al clima hiper-evaluativo instaurado desde su implementación. 


\section{El Programa de Incentivos y sus notas: el docente $i$ "atrapado sin salida"?}

El Programa se denomina de esta manera porque introduce un incentivo económico o pago adicional para mejorar el ingreso (sueldo) de aquellos docentes que han obtenido una categoría de docente investigador, requisito necesario para incorporarse a él. Al respecto sostiene que:

[...] la creación de un incentivo a los docentes de las Universidades Nacionales que participen en proyectos de investigación contribuirá en forma simultánea a aumentar las tareas de investigación y desarrollo a nivel nacional, fomentar la reconversión de la planta docente, motivando una mayor dedicación a la actividad universitaria y a la creación de grupos de investigación. (MINISTERIO DE EDUCACIÓN, 1993).

El cobro de este incentivo impone aceptar condiciones ineludibles. Está supeditado al cumplimiento de tareas de enseñanza y de investigación debidamente verificadas y certificadas por autoridad competente y al resultado de la evaluación periódica de las actividades de investigación y de los currículos de los docentes participantes.

Para la presentación de los datos del desempeño académico, cuenta con un instrumento evaluativo de marcado carácter descriptivo que discrimina en diversos apartados las actividades exigidas al docente-investigador: "Grilla para evaluación de antecedentes para categorización en el programa de incentivos". Se trata de un dispositivo nodal para la valoración de la actuación y productividad individual. En el marco de periódicas Convocatorias de Categorización instituidas por el Programa, los Pares Evaluadores designados para tal fin, basándose en la suma de los puntajes parciales de los nueve rubros que componen esta Grilla, conceden o niegan la categoría solicitada por cada docente, quien puede aspirar a una de las cinco vigentes (I, II, III, IV y V) según obtenga un puntaje de 1100, 750, 500, 300 o 100 respectivamente. (MINISTERIO DE EDUCACIÓN, 2008).

A hora bien, cifras divulgadas por la Secretaría de Políticas Universitarias (SPU) informan que el Programa de Incentivos - que inició su actividad incorporando 7.961 docentes - cuenta en la actualidad con 32.000 docentes-investigadores concentrados mayoritariamente en las áreas de las ciencias naturales, exactas y sociales y que ha ampliado en un $23 \%$ la base de la estructura de investigación fortaleciendo la inclusión y la formación de jóvenes docentes investigadores. 
Frente a estas cifras y ante la resistencia de muchos docentes de someterse a sus imposiciones, vale preguntarse, ¿por qué se involucran en él si su ingreso es voluntario?. En realidad, su carácter opcional conlleva paradojalmente una necesaria obligatoriedad. Se trataría más bien de un estar atrapado sin salida, al decir de una colega, de una falsa ilusión de decisión personal por parte del docente, ya que la renuncia a sus reglas de juego pone en riesgo la permanencia / promoción de su propio cargo y la autoexclusión de la dinámica de actividades que atraviesan el quehacer cotidiano de las unidades académicas, de códigos discursivos entre colegas y del público reconocimiento que el mundo universitario otorga a las categorías asignadas por los pares evaluadores. (VILLAGRA; CASAS, 2010).

Escuchemos una representativa voz docente:

[...] El programa de incentivos existe y existirá ... ¿ ¿mantenerte al margen? Una utopía que uno sueña cuando te sentís agobiado por sus tantísimas exigencias. Ni plantearse el desconocerlo porque sí o sí, tengas o no tiempo, coincidas o no coincidas con sus fundamentos y forma de funcionar, tu cargo depende de que puedas, como sea, mantenerte adentro de él. Es así... Si no estás categorizado tus colegas de cátedra y Facultad te ven como un don nadie... como si no existieras... uno mismo termina sintiéndose así.

Constatamos, entonces, que la aceptación de la normativa del Programa de Incentivos no significa necesariamente una genuina adhesión. Estar o no estar en el sistema, que no es lo mismo que estar o no estar con el sistema, supone la presencia de contradicciones que convocan sensaciones, emociones, actitudes y pensamientos antagónicos muy difíciles de conciliar, afectando la calidad del desempeño docente, el placer o displacer por la tarea y la modalidad de vínculo con la enseñanza, con los estudiantes y fundamentalmente con el conocimiento.

\section{La instauración de un clima hiper-evaluativo ocupa la escena}

El tratamiento de la problemática de la intensificación laboral requiere ser situada en el clima institucional que marca/demarca los ritmos de trabajo del docente, en tanto condiciones objetivas que afectan su subjetividad. Un rasgo hegemónico asume indudable relevancia: en el contexto de las políticas de eva- 
luación imperantes, todo se evalúa: universidades, facultades, carreras de grado y de posgrado y funciones académicas anudadas en el desempeño docente. Es en este espacio donde el Programa de Incentivos ocupa la escena. Varias son las instancias evaluativas formales sobre las que se sostiene este Programa. Los informes anuales de avance de los proyectos de investigación - WinSip - dibujan itinerarios de trabajo individual y/o grupal que delatan pública y privadamente grados de productividad/improductividad. El Director pondera la actividad de los integrantes de su equipo, a la vez, evaluadores externos valoran la actuación del Director y lo generado en el proyecto bajo su gestión y cada integrante, ineludiblemente, se evalúa a sí mismo.

El interjuego entre la hetero y la autoevaluación se transversaliza, impregnando la vida universitaria. Las acciones académicas valen, suman y/o restan instaurando un clima hiper-evaluativo que prioriza lo cuantitativo sobre lo cualitativo, la competitividad sobre la solidaridad, el control de lo actuado sobre la libertad de iniciativa. La naturalización de la lógica de la rendición de cuentas o accountability, introduce así nuevos modos de convivencia entre colegas, reestructura la configuración de las cátedras y su microclima de producción, generando cambios sustanciales en las culturas académicas y una redefinición identitaria del docente universitario.

He aquí un testimonio:

[...] En mi trabajo respiro evaluación, sueño con la evaluación ... que ya llega el nuevo Win Sip, que tengo que inspirarme en el llenado de mi grilla para que me den los puntajes, que necesito más ponencias en congresos y este año no pude viajar, que la próxima reunión con la comisión de seguimiento de mi tesis estará durísima, que se hará el llamado concurso de mi cargo, que qué y más que... todo, todo esto en un tiempo que no alcanza... ni alcanzo...

Párrafo aparte lo que detona el presentarse a categorización. Constituye un acto tan determinante como memorable para la biografía académica. La Grilla de Categorización obliga a una precisa y exhaustiva revisión de lo hecho-no hecho, de lo documentado y de lo indocumentado (no probado en papeles), que deriva generalmente en una culposa reactualización de lo queda por hacer. Cada rubro de la Grilla devuelve, desde la ausencia o presencia de datos pertinentes, antecedentes consolidados en algunas áreas, débiles en otras y/o el ostensible vacío de acciones en algunas dimensiones. El proceso de su completamiento pareciera aproximarse más a una operación matemática que a una sistematización 
de lo actuado en una trayectoria académica ya que, sustentado en criterios de evaluación sumativa y tal como ya lo señalamos, obliga obsesivamente a computar resultados. El Curriculum Vitae materializado en el formato de la Grilla visibiliza, a la manera de espejo, una imagen coincidente o contrapuesta de la que cada docente construye de sí mismo. Esta mirada no exenta de resistencia, en muchos casos lleva al docente a través de un relectura de las certificaciones de trabajo acumuladas, a un forzado reacomodamiento de sus datos en la búsqueda de la obtención de un puntaje más elevado.

\section{La intensificación del trabajo docente o la falta de tiempo como megapretexto}

Según conceptos inherentes a la ciencia económica, la intensificación se define como la producción de más unidades de trabajo en la misma unidad de tiempo. (DAVINI, 1995). La intensificación del trabajo docente consiste, entonces, en el aumento de la cantidad de obligaciones dentro de una misma carga horaria.

La convencional centración del docente en la transmisión de conocimientos cobra fuerza expansiva, según lo expusimos, a partir del Programa de Incentivos debido a que la enseñanza debe complementarse con la investigación, la extensión y la gestión, con un agregado que remarcamos: todas serán acreditadas. El incremento de obligaciones al anudarse con la evaluación conforma un bloque compacto de cantidad-evaluada cuyo peso suscita una vivencia de sobrecarga laboral sentida por momentos como insostenible, posicionando al docente en un lugar de vulnerabilidad.

La intensificación toma la forma de un repertorio siempre abierto de heterogéneas tareas que esperan ser saldadas. Cuáles, cuándo, cómo o con quien/ es abordarlas, requiere constantemente definir tiempos, espacios y prioridades. Sin embargo, y a pesar de intentos organizativos individuales y/o grupales, las acciones son tantas y de naturaleza tan diferente, que frecuentemente se yuxtaponen entrelazándose en tiempos simultáneos.

[...] Por momentos no sé por dónde iniciar ni por dónde seguir; todo se me acumula. En este cuatrimestre tengo que ocuparme de la cátedra $y$ del "voluntariado" y sé que me convendría presentar trabajos en dos importantes encuentros, muy exigentes en la aprobación de los "abstracts", lo que me insume mucho tiempo. Porque me cuesta escribir. 
Desgraciadamente no tengo con quién hacer la ponencia... mis colegas están con sus posgrados. Y eso que no quiero acordarme de concursos por tomar y de... bueno... siempre me pasa lo mismo.

En la expresión más en menos tiempo, que imbrica lo cuantitativo con lo temporal, radicaría la clave que justifica en la información procesada la protagónica presencia de una queja: la falta de tiempo. Por su obstinada reiteración convenimos en designarla como megaqueja o megapretexto, reconociéndola como un rasgo identitario del actual discurso docente. Esta megaqueja se apropia del sentido de lo que pertrecha, de lo que protege ante el atosigamiento de demandas del sistema (VILLAGRA; CASAS, 2010) erigiéndose como un generalizado objeto de proyección de aquéllo que no se puede, que no se intenta o que no satisface. No descartamos, sin embargo, que en muchos casos su uso y abuso encubriría cierta victimización y/o ausencia de autocrítica.

Ahora bien, las colisiones entre el incremento de impostergables tareas y de "...un tiempo que no alcanza... ni alcanzo" (expresión docente ya transcripta) resignifican desde un desafiante replanteo un clásico tópico pedagógico: ¿calidad versus cantidad?. Los registros analizados identifican la adopción por parte de algunos docentes de una preocupante actitud no exenta de malestar o de resignada impotencia: hacer lo que se debe, no siempre coincidente con lo que se quiere ni cuándo ni cómo se lo desea, conduce a una sensación residual de un hacer sólo como se puede. Lo cierto es que la coalición entre lo urgente y lo requerido, enmarcada por la presión de una hiper-evaluación real y/o simbólica de lo exigido, entablan una natural complicidad con la cantidad de tareas, lejos de propiciar un cuestionamiento de la calidad de las mismas. (VILLAGRA, 2011).

[...] Si intento hacer todo como desearía no hago nada, entonces me digo, hay que hacer como pueda y cuando se pueda. Es fatal exigirte tanto ante tanta sobrecarga... Así pensamos en mi cátedra porque estamos convencidos que se premia la cantidad... eso de que a mayor calidad menos cantidad y más tiempo, ya lo sabemos y el reloj corre...

\section{De deudas pendientes a una colonización de los ámbitos privados del docente}

Como configurante de la intensificación del trabajo, aparece un elemento muy perturbador para el docente: lo que aún falta, lo pendiente. Por un inevitable 
efecto acumulativo, y casi imperceptiblemente, se pluraliza en un listado de lo que sigue restando en referencia a actividades y producciones que se postergan indefinidamente en el tiempo. Irrumpe así la vivencia de deuda, de una cantidad de cosas que se debe y que se está obligado a saldar y deviene entonces lo que denominamos estado de endeudamiento crónico. Se caracteriza, a la manera de un pago diferido, por implicar una prolongada y tensa duración que desde un tiempo fenomenológico moviliza vivencias de atemporalidad y que, tal como lo inferimos de entrevistas en profundidad, genera reacciones extremas: desde una inacción casi paralizante ante lo adeudado hasta una ansiógena hiperactividad que confunde lo necesario con lo posible, lo importante con lo accesorio.

[...] Cuando más cosas pendientes tengo, me parece que más me bloqueo. No puedo hacer ni pensar nada ... es como si el tiempo se detuviera y no lo pudiera mover, paralizando todo lo que tengo postregado. Es una rara sensación de aplastamiento y de imposibilidad... mi excusa es que algún día podré, día que no llega nunca. Me pasa al revés que otros colegas que no pueden parar, que pasan de una actividad a otra, como si no pudieran "cortar"...

En el material procesado advertimos que el soportar (o no) lo que se adeuda - vivido en realidad como una deuda para sí mismo - constituye uno de los rasgos más angustiantes y difíciles de metabolizar por el docente. Este autoadeudamiento se potencia sobremanera frente a los dispositivos de evaluación de la productividad, especialmente ante la Grilla de Categorización. La ausencia de datos en algunos rubros, la insuficiencia o la dudosa inclusión en otros, convoca temores, nostalgias y una íntima disconformidad. Reactualiza y sobredimensiona lo que no se pudo hacer viviéndoselo como atentatorio y culposo en relación con la imagen que el docente intenta sostener de sí mismo. La distancia cercana y/o lejana entre lo que se es, se creía ser y lo que se desearía ser, provoca una fuerte movilización de orden subjetivo en donde la pugna entre el auto-reconocimiento y el hetero-reconocimiento, entre una autopercepción y un yo que se desconoce, lleva hasta poner bajo sospecha la objetividad de las evaluaciones $\mathrm{y} / \mathrm{o}$ de los evaluadores.

Según comprobamos en nuestros avances investigativos, este estado de endeudamiento crónico avanza gradualmente hacia una colonización de los tiempos y de los espacios personales-profesionales. Este concepto de colonización, inserto dentro de la dimensión sociopolítica del tiempo (HARGREAVES, 1998, p. 135-136), ha cobrado una especial relevancia en el mundo actual de 
la docencia universitaria. Tiene lugar cuando el cumplimiento del incremento de tareas decretadas traspasa los límites espacio-temporales que demarcan el territorio de lo laboral y se apropia o invade áreas cerradas o de trastienda del trabajo docente (espacios privados e informales). La vida familiar, el ocio, actividades extralaborales comienzan a posponerse o borrarse para ser habitadas por el trabajo. Ante esta tensión entre lo público y lo privado, entre lo laboral y lo extralaboral siempre propensa a una posible fusión y/o confusión, el docente claudica o se resiste.

La claudicación puede llevar simbólicamente al docente a una posible fagocitación de su vida personal por el trabajo académico, con resonancias de fragilización psicofísica. En muchos discursos analizados, llamativamente se deslizan síntomas que ponen en palabras una insatisfacción generalizada. La explicitación de cansancio y fatiga psicológica y orgánica, de pérdida de motivación por el trabajo, de sentimientos de fracaso, de baja realización profesional $\mathrm{y} / \mathrm{o}$ personal, de imposibilidad de soportar presiones institucionales, entre otros (GUERRERO BARONA, 2003) pueblan más de lo deseado las impresiones que refieren al trabajo docente.

En el análisis de los datos puede advertirse que en aquellos docentes que se resisten a esta colonización y asumen la defensa de zonas existenciales privacidas, cobra notoria preeminencia un resguardo: el cálculo diferenciado de los insumos temporales según el tipo de actividad académica. Como el tiempo vale - escasea - se agota, el proceso de dosificación / distribución tendiente a la evitación de su malgasto, requiere la adopción de racionales previsiones en torno a su inversión. Al respecto, puede constatarse una reiterada elección: los insumos, regidos por las leyes de la rendición de cuentas, se orientan predominantemente hacia aquellas actividades más valoradas cuantitativamente y que privilegian la consolidación de su profesionalidad. Indudablemente, entonces, hacia la investigación.

\section{¿Hacia un desdibujamiento de la enseñanza?}

A esta altura un se impone un interrogante ¿Qué posición ocupan en los actuales procesos de reforma las prácticas de enseñanza o la docencia propiamente dicha? Los avances de la investigación dan cuenta de un reposicionamiento del lugar de la enseñanza.

La arraigada modalidad de ser docente anclada en la enseñanza de una o más asignaturas como proyecto compartido cotidianamente con colegas de 
cátedra y estudiantes, se ha desdibujado sensiblemente. Puede interpretarse que ya se preanunciaba tal trastrocamiento desde la inocultable y explícita centralidad otorgada a la investigación por el Programa de Incentivos. Ahora lo estamos comprobando.

El avance de la naturalizada actitud especuladora de priorizar ciertas acciones académicas sobre otras, por ser más redituables en términos de los puntajes asignados, desoculta la introducción de lógicas de actuación que afectan visceralmente el vínculo con la enseñanza universitaria y por ende con el conocimiento. Si estamos asistiendo a una provocativa claudicación de lo que se prefiere por lo que conviene, esta conveniencia ¿se vincula con la docencia?

La Grilla de Categorización responde crudamente a esta pregunta. Basta revisarla para comprobar que subestima su tradicional valía en relación a la premiada sobrevaloración de otras actividades. Los componentes que conforman su apartado, develan cómo es visibilizada. Interesan los tipos de cargos docentes $y$ de publicaciones, la actuación en carreras de posgrado, la integración de tribunales de concursos y/o tesis de posgrado, la dirección de trabajos finales $y$ de pasantes y la integración de comisiones evaluadoras. Indudablente se solapan acciones didácticas neurálgicas y potentes, provocadoras de innovaciones pedagógicas. Al ser reubicadas detrás del telón, signadas por el anonimato se invisibilizan como objeto de evaluación. Actividades cotidianas propias de una buena enseñanza tales como el contacto con los estudiantes, las discusiones temáticas, las reuniones de cátedras e intercátedras, el diseño de evaluaciones más reflexivas, el ensayo de nuevas estrategias de enseñanza, entre otras acciones que los puntajes des-conocen, ceden ante las recompensadas tareas de investigación y van retirándose de las escenas de aula. Lejos de prácticas ineludibles, han comenzado a ser percibidas como excepcionales y en tanto no computables, se subvaloran como residuales por tratarse de acciones que no suman sino restan en los puntajes que dictaminan el status docente. La docencia se ubicaría así en una zona de borde, tornándose visible su situación de riesgo.

En consonancia con esta línea de planteos, una especialista argentina en problemáticas de educación superior universitaria expresa que

[...] en las situaciones en las que se someten a evaluación con el propósito de entrar o ascender en el sistema, también aprenden que las identidades individuales construídas sobre la base de otras actividades en su historia profesional, tales como la enseñanza y la extensión, ya no tiene el valor de antaño. (ARAUJO, 2002, p. 248). 


\section{Formas deformadas de actuación o formas defensivas de reaseguro laboral}

Del procesamiento de la información obtenida en nuestra investigación pudimos aislar un impactante hallazgo: la entrada en escena de mecanismos de acomodación y/o resistencia desplegados por los docentes para soportar o reparar sensaciones de impotencia o incompetencia ante la presión de las exigencias del Programa de Incentivos. La obligada adaptación a su normativa incitó a un despliegue de actitudes de impostura o simulación cuya expansión resulta preocupante. En entrevistas en profundidad pudo corroborarse que el actuar desde el como si o cuestionable construcción de la apariencia posibilitó al docente investirse de un parecer habilitante de la ilusoria sensación de seguir siendo un docente con perfil académico aceptado.

Psicológicamente resulta entendible que todo cuestionamiento a la identidad profesional implique un menoscabo a la identidad personal ya que en el plano de lo imaginario la no superación de las exigencias explícitas e implícitas del Programa de Incentivos reactualiza ansiedades básicas que afectarían destructivamente la configuración del yo. Es indudable que a nivel de la dimensión emocional se produce un impacto en el narcisimo y en la disminución de la autoestima que lleva en muchos casos a que el malestar docente se exprese como una necesidad de castigo.

La circulación de una serie de formas deformadas de actuación ya conocidas en el contexto académico se potenciarían por la política de incentivos. (ARAUJO, 2002, p. 248). Esta autora sostiene que ha promovido mecanismos fraudulentos en la educación en general y particularmente en la investigación (el plagio, el autoplagio, la fabricación de datos, otros) y ha contribuido a la instauración de modos de supervivencia tales como la compulsión a la producción-publicación sin interesar su originalidad y calidad; la coautoría múltiple, la presencia de autores fantasma, el envío de trabajos sin asistencia a las reuniones científicas, el armado del curriculum vitae en función de requisitos de evaluación, para citar sólo algunos.

Voces docentes atestiguan posturas antagónicas: "bueno... adaptado no estoy... sólo parece, pero en el fondo todavía me resisto, hay formas de permanecer que dan resultados pero que rotundamente no acepto..."; "la cuestión de los antecedentes tiene y no tiene que ver con la calidad de tu trabajo... se trata de saber presentarlos, es decir, saber decirlos, "venderlos" y no solamente hacerlos... son modos de susbsistir".

Nos interesa enfatizar que estos mecanismos y modos de supervivencia, también explicables como formas defensivas de reaseguro económico y/o 
psicológico (preservar el prestigio, la autoestima, el propio nombre) en tanto requieren un considerable insumo de energía, acentúan la descentración del docente respecto a las acciones de aula, de enseñanza.

Sin duda, lo más inquietante radica en la gradual naturalización de la adopción de estas estrategias por parte de los miembros de la comunidad docente y en la progresiva desnaturalización de valores y prácticas académicas históricamente privilegiadas y éticamente incuestionables en la universidad.

\section{Un cierre abierto a nuevos replanteos}

El impacto de las Reformas de la Educación Superior en Argentina indudablemente ha re-formado el Trabajo Docente. Desde una mirada cualitativa intentamos rescatar flashes de lo que acontece actualmente en su ámbito laboral, pantallazos que devuelven una visión de cómo los docentes viven y/o sobreviven la reescritura de su función, la búsqueda de una nueva forma de ejercerla, si no todos, al menos muchos de ellos. Tal como apuntamos, alerta la desnaturalización de valores y prácticas académicas históricamente privilegiadas y éticamente incuestionables a causa de la naturalización de actuaciones docentes, si no contrarias, al menos atentatorias a ellos. El contexto hiper-evaluativo ya dejó sus huellas en las culturas académicas y en sus integrantes que se abaten y debaten ante la intensificación de tareas, el endeudamiento crónico frente a ellas y la rendición de cuentas.

De allí que no es casual que el contenido de este texto, inspirado en los decires de profesionales argentinos, haya permitido repasar peligros derivados de la implementación de lógicas de evaluación con la intencionalidad de dejar abiertos al debate alertas para repensar.

A manera de síntesis, quedan a consideración:

- En general ejercen un papel disciplinador indicando qué debe hacerse, "voluntaria y libremente" para poder sumar puntos al expediente, y en qué no vale la pena utilizar esfuerzos porque no está puntuado.

- Genera una ciudadanía “domesticada”, cuyo núcleo de acción está puesto en la adaptación y en el cumplimiento, aparente o real, de lo prescrito.

- Impulsa conductas de simulación y fuerte burocratización de los procesos.

- Alienta el individualismo en la construcción de un curriculum de antecedentes por la lógica intrínseca de ganar mejores puestos en el ranking. 
- Desalienta actitudes de indagación crítica acerca de temas globales de la educación y a sociedad, puesto que, al no figurar en los instrumentos de evaluación, enseña que ésos, no son temas de la incumbencia de los profesores. (CELMAN, 2000).

\section{REFERENCIAS}

ARAUJO, S. Evaluación, incentivos a la actividad investigadora y trabajo académico. Algunas conclusiones en el estudio de un caso en la Argentina. En: KROTSCH, P. La universidad cautiva. Legados, marcas y horizontes. Buenos Aires: Al Margen, 2002. p. 231-251.

CELMAN, S. Las políticas de evaluación docente en el marco de las Reformas Educativas de los 90: un encuadre desde el cual interrogar la nueva etapa. En: CONGRESO INTERNACIONAL DE EDUCACIÓN, II., 2000, Buenos Aires. Anales... Buenos Aires: Universidad de Buenos Aires, 2000.

DAVINI, M. C. La formación docente en cuestión: política y pedagogía. Buenos Aires: Paidós, 1995.

GUERRERO BARONA, E. Análisis pormenorizado de los grados de burnout y técnicas de afrontamiento del estrés docente en profesorado universitario. Anales de Psicología, Murcia: Servicio de Publicaciones de la Universidad de Murcia, v. 19, n. 1, p. 145-158, jun. 2003.

HARGREAVES, A. Profesorado, cultura y postmodernidad: cambian los tiempos, cambia el profesorado. Madrid: Morata, 1998.

MINISTERIO DE EDUCACIÓN. Secretaría de Políticas Universitarias. Programa de Incentivos a Docentes Investigadores. Decreto n 2427. Buenos Aires, 1993.

MINISTERIO DE EDUCACIÓN. Secretaría de Políticas Universitarias. Categorización Cuarta Convocatoria. Pautas de Evaluación. Buenos Aires, 2008.

VILLAGRA, A. Docencia universitaria e intensificación de prácticas académicas: una lectura desde lo espaciotemporal. En: JORNADAS NACIONALES SOBRE LA FORMACIÓN DEL PROFESORADO, VI., 2011, Mar del Plata. Anales... Universidad Mar del Plata: Nacional de Mar del Plata, 2011.

VILLAGRA, A.; CASAS. N. Políticas académicas argentinas y trabajo docente universitario. En: CONGRESO IBEROAMERICANO DE DOCENCIA UNIVERSITARIA, VI., 2010, Lima. Anales... Lima: Pontificia Universidad Católica del Perú, 2010.

Texto recebido em 12 de agosto de 2015. Texto aprovado em 12 de agosto de 2015. 
